The Solution of the Metric STRESS and SSTRESS Problems in Multidimensional Scaling Using Newton's Method

\author{
A.J. Kearsley \\ R.A. Tapia \\ M.W. Trosset
}

December 1994

TR94-44 



\title{
The Solution of the Metric STRESS and SSTRESS Problems in Multidimensional Scaling Using Newton's Method
}

\author{
Anthony J. Kearsley* Richard A. Tapia ${ }^{\dagger} \quad$ Michael W. Trosset ${ }^{\ddagger}$ \\ January 8, 1995
}

\begin{abstract}
This paper considers numerical algorithms for finding local minimizers of metric multidimensional scaling problems. The two most common optimality criteria (STRESS and SSTRESS) are considered, the leading algorithms for each are carefully explicated, and a new algorithm is proposed. The new algorithm is based on Newton's method and relies on a parametrization that has not previously been used in multidimensional scaling algorithms. In contrast to previous algorithms, a very pleasant feature of the new algorithm is that it can be used with either the STRESS or the SSTRESS criterion. Numerical results are presented for the metric STRESS problem. These results are quite satisfying and, among other things, suggest that the well-known SMACOF-I algorithm tends to stop prematurely.
\end{abstract}

Key words: Metric multidimensional scaling, STRESS criterion, SSTRESS criterion, unconstrained optimization, Newton's method, SMACOF-I.

\footnotetext{
*Department of Computational and Applied Mathematics, Rice University, Houston, TX 77251-1892. This author was supported by a Patricia R. Harris Fellowship and a Center for Research in Parallel Computation Graduate Fellowship.

†Department of Computational and Applied Mathematics and Center for Research in Parallel Computation, Rice University. This author was supported in part by NSF Coop. Agr. No. CCR-8809615, AFOSR 89-0363, and DOE DEFG05-86ER25017.

$\ddagger$ Department of Computational and Applied Mathematics (adjunct), Rice University; Department of Psychology (adjunct), University of Arizona; and Consultant, P. O. Box 40993, Tucson, AZ 85717-0993. This author was supported in part by NSF Coop. Agr. No. CCR-8809615, as a visiting inember of the Center for Research in Parallel Computation, Rice University, August 1993 and 1994.
} 



\section{Introduction}

Multidimensional scaling (MDS) is a general term for a vast collection of data analytic techniques. As defined by de Leeuw and Heiser [11], scaling refers to techniques that construct a configuration of points in a target metric space from information about interpoint distances, and MDS is scaling in the case that the target space is Euclidean. Kruskal and Wish [29] provided an elementary introduction to basic MDS methodology, as well as many enlightening examples.

The present paper addresses two very specific, but very important problems in MDS. As in classical MDS [42, 43, 19], two assumptions are made about the nature of the information provided about the interpoint distances. Formally, a symmetric $n \times n$ matrix $\Delta=\left(\delta_{i j}\right)$ is called a dissimilarity matrix if $\delta_{i j} \geq 0$ (nonnegative elements) and $\delta_{i i}=0$ (zero diagonal elements). From a given dissimilarity matrix $\Delta$, a two-way MDS algorithm constructs a configuration of points in a Euclidean space of specified dimension $p$. For a configuration $x_{1}, \ldots, x_{n} \in R^{p}$, the $n \times p$ configuration matrix $X$ is the matrix whose rows are the $x_{i}^{T}, i=1, \ldots, n$. From $X$ it is easy to compute the Euclidean interpoint distance matrix $D(X)=\left(d_{i j}\right)$. The objective of two-way MDS is to construct a configuration for which the interpoint distances $d_{i j}$ somehow approximate the given dissimilarities $\delta_{i j}$.

To each possible configuration corresponds a matrix of interpoint distances. Historically, MDS techniques that minimize some measure of discrepancy between the set of interpoint distance matrices and the given dissimilarity matrix $\Delta$ have been termed metric. In contrast, nonmetric techniques minimize some measure of discrepancy between the set of interpoint distance matrices and a set of dissimilarity matrices whose elements have the same rank ordering as the given $\delta_{i j}$. Early MDS techniques, e.g. the methods of Torgerson [42], were exclusively metric. However, since the pioneering work of Shepard [38,37] and Kruskal [27, 28], the psychometric and statistical communities have tended to emphasize nonmetric MDS. Nevertheless, metric MDS has remained critically important, because solving nonmetric MDS problems typically involves repeatedly solving metric MDS subproblems. In recent years, there has been renewed interest, e.g. by de Leeuw [8], in developing efficient methods for solving these subproblems. Furthermore, in the last decade, techniques related to MDS have been studied by computational chemists, e.g. Crippen and Havel [6], interested in deducing molecular structure from information about interatomic distances. Because procedures such as NMR spectroscopy do not distort distances in the nonlinear ways that human perceptions of psychophysical phenomena typically do, it is metric MDS that is of interest in this context. Thus, the study of metric MDS remains of fundamental importance.

The classical metric approach of Torgerson [42] having fallen from favor, most modern formulations of metric MDS entail the minimization of one of two measures of the discrepancy between distances and dissimilarities. The STRESS criterion, proposed by Kruskal [27] for nonmetric MDS, is based on the squared errors between the distances and the dissimilarities. The SSTRESS criterion, popularized by Takane, Young, and de Leeuw [40] for nonmetric MDS, is based on the squared errors between the squared distances and the squared dissimilarities. Thus, both the metric STRESS $(r=1 / 2)$ and SSTRESS $(r=1)$ problems are special cases of the following constrained optimization problem:

$$
\begin{array}{ll}
\text { minimize } & \sum_{i<j} w_{i j}\left[\left(d_{i j}^{2}\right)^{r}-\left(\delta_{i j}^{2}\right)^{r}\right]^{2} \\
\text { subject to } & D \in \mathcal{D}_{n}(p),
\end{array}
$$

where the $w_{i j}$ are nonnegative weights and $\mathcal{D}_{n}(p)$ is the set of all $n \times n$ matrices whose elements can be realized as the interpoint distances of $n$ points in $R^{p}$. In practice, one often sets each $w_{i j}=1$; however, one can use the weights either to accomodate missing data (by setting the appropriate $w_{i j}=0$ ) or to weight more reliably measured dissimilarities more heavily. In most applications, the dimension of the configuration space is small; in the case of molecular conformation, of course, one always sets $p=3$.

The purpose of the present paper is to describe an implementation of Newton's method for the efficient solution of Problem (1) in the special cases $r=1$ and $r=1 / 2$. In Section 2 we discuss several fundamental concepts in metric MDS and numerical analysis. This section provides the necessary background for our review of the leading metric MDS algorithms (Section 3) and for our description of a more efficient algorithm (Section 4). We present numerical results in Section 5 and assess what we have accomplished in Section 6. 


\section{General Considerations}

Because it is not obvious how to write the constraint $D \in \mathcal{D}_{n}(p)$ in Problem (1) as standard equality or inequality constraints, it cannot be managed by the standard techniques of mathematical programming. Therefore, virtually all treatments of MDS problems employing either STRESS or SSTRESS parametrize the distances by expressing them in terms of the configuration coordinates, i.e. by writing

$$
d_{i j}^{2}=d_{i j}^{2}(X)=\sum_{k=1}^{p}\left(x_{i k}-x_{j k}\right)^{2} .
$$

Substituting (2) into (1) eliminates the constraint but complicates the objective function, resulting in the unconstrained optimization problem

$$
\operatorname{minimize} \quad \sum_{i<j} w_{i j}\left[\left(\sum_{k=1}^{p}\left(x_{i k}-x_{j k}\right)^{2}\right)^{r}-\left(\delta_{i j}^{2}\right)^{r}\right]^{2} .
$$

Henceforth, we restrict attention to this parametrization of the metric STRESS and SSTRESS problems.

The remainder of this section discusses several fundamental issues from the theory and practice of unconstrained optimization that are germane to the efficient solution of Problem (3). We believe that an appreciation of these issues is essential to our critique of previous algorithms (Section 3) and our presentation of a new algorithm (Section 4). More detailed discussions of these issues can be found in the well-known book by Dennis and Schnabel [13].

Let us begin with a comment about the distinction between local and global minimizers. Ideally, we would like to find a global minimizer of Problem (3). Unfortunately, whereas the science of local optimization is highly advanced, the science of global optimization is still in its infancy. To date, all of the important methods proposed for metric MDS have been iterative algorithms for finding local minimizers. In this paper, we are content to improve on these algorithms. Typically, one attempts to find a "good" local minimizer by finding a good initial configuration from which to start iterating. It has also been suggested that global minimizers might be more readily obtained by exploiting the geometry of the cone of distance matrices (Glunt, Hayden, and Liu [16] demonstrated that all of the local minimizers of the metric SSTRESS problem lie on the surface of a sphere, the global minimizer being the one of greatest norm) or by parametrizing the configuration in a larger (than $p$ ) number of dimensions. Thus far, effective algorithms based on these ideas have not been forthcoming. Finally, several researchers have applied global optimization methods to the metric STRESS problem. The modularized DG-II package of Havel [22] attempts to improve on a local minimizer by the use of simulated annealing. A detailed study by Groenen [20] suggests that tunneling methods are preferable to simulated annealing.

In the modern theory of computational optimization, Newton's method continues to be the method of choice for finding local solutions of unconstrained optimization problems. Under well-known standard assumptions about smoothness and nonsingularity, it is not difficult to establish local and fast (quadratic) convergence of the method. Critics of Newton's method direct their comments to the need for calculating second derivatives, the need for solving a linear system of equations at each iteration, the implied need for smoothness and nonsingularity, and the implied nonglobal convergence. While the general theory is sharp, and all of the above may be valid criticisms across the full spectrum of applications, experience has shown that, in a particular application, not all of these criticisms need apply or be restrictive. What is at issue in

the present paper is the applicability of these criticisms to the metric STRESS and SSTRESS problems of MDS.

Users of optimization algorithms are often (and understandably) confused by the distinction between local and global convergence. What is at issue here is not the type of minimizer, but where the algorithm must start in order that convergence to a local minimizer be guaranteed. Theory tells us that, if Newton's method starts sufficiently near an isolated local solution, then the sequence of iterates will rapidly converge to that solution. However, this property does not preclude the possibility of choosing a starting point from which the sequence of iterates may fail to converge to any local minimizer. Modifications of locally convergent methods that eliminate this undesirable possibility are called globalization strategies, and algorithms that are guaranteed to converge to a local minimizer from (essentially) every starting point are said to be globally convergent. 
Actually, decades of experience have demonstrated that the semi-local properties of Newton's method are usually quite good - much better, in fact, than the local theory predicts. Convergence and fast convergence are usually not restricted to very small neighborhoods of solutions, as many vendors of awkward hybrid methods would have us believe. Consider that any superlinearly convergent algorithm gives arbitrarily good linear decrease in error in neighborhoods of solutions. Unless the objective function is highly nonlinear, these neighborhoods may be quite large. Hence, Newton's method can be particularly effective for optimizing mildly nonlinear functions by virtue of exhibiting very fast linear convergence in large neighborhoods of solutions. Nevertheless, Newton's method per se is not globally convergent. Two fundamental globalization strategies for Newton's method, line searches and trust regions, are discussed in Chapter 6 of Dennis and Schnabel [13]. Both are based on the idea that each step taken by the algorithm should decrease the value of the objective function.

Extensive experience with Newton's method has also demonstrated that damping the Newton step, i.e. choosing step length less than one, often improves the global behavior of Newton's method. However, not choosing step length one locally may preclude the fast convergence. Concern for these two aspects of Newton's method has resulted in the so-called backtracking line-search strategies, in which one always considers the full Newton step before damping, and one implements damping in a manner that ensures the full Newton step near the solution. It should also be noted that many unconstrained optimization algorithms allow steps of length greater than one.

Line-search strategies retain the Newton step direction but alter its length. In contrast, trust-region strategies constrain the step length but allow other choices of the step direction. This is accomplished by searching for steps that minimize a quadratic approximation to the objective function at the current iterate, subject to an upper bound on the step length. Because there is no finite way of exactly solving this quadratic subproblem, various approximate solutions have been suggested. The most commonly used are the "hook" step of Hebden [23] and Moré [32], and the "double dogleg" step due to Powell [35].

In general, the presence of a singular Hessian matrix at a solution dramatically slows - and may even preclude - the local convergence of Newton's method. Moreover, if the solutions are not isolated, then the Hessian matrix is necessarily singular at a solution. For this reason, we prefer problem formulations that yield isolated solutions. Notice, however, that the objective function in Problem 3 is invariant under isometric transformations of the configuration, so that every minimizer belongs to a connected set of minimizers. What has happened is that the reparametrization from (1) to (3) introduced a considerable amount of redundancy. To develop an efficient algorithm for solving Problem (3), it is desirable to remove this redundancy. As we shall see in Section 3, different researchers have addressed this need in different ways.

Finally, we consider the computational issues of calculating second derivatives and solving systems of linear equations at each iteration of Newton's method. A critical issue in deciding to use the method is the viability of performing these computations. It is certainly naive to believe that this viability can be determined by looking at only one iteration. Rather, the complete picture must be considered. Discarding Newton's method in favor of an algorithm that produces cheap iterates is of no value if the number of iterations needed to solve the problem is prohibitively large. This is often the case for gradient methods, unless only a very limited amount of accuracy is needed. It follows that whether or not Newton's method will be successsful in a particular application can only be decided by careful study of the application, in conjunction with careful numerical experimentation.

\section{Previous Algorithms}

We now review the most important of the algorithms that have been proposed for metric MDS. Historically, researchers have developed completely different algorithms for the metric STRESS and SSTRESS problems. In contrast, we believe that one of the attractive features of our approach is that we have developed a single algorithm that works well on both problems.

Our primary emphasis in this section is on the numerical algorithms that have been used to generate sequences of iterates. However, we are also concerned with the approaches that different researchers have taken to isolate local minimizers, and with the devices that they have used to obtain starting points for their algorithms. 


\subsection{STRESS}

The STRESS criterion is the objective function in Problem (3) obtained by setting $r=1 / 2$. Let us write the STRESS criterion as

$$
\sigma(X)=\sum_{i<j} w_{i j}\left[\left(\sum_{k=1}^{p}\left(x_{i k}-x_{j k}\right)^{2}\right)^{1 / 2}-\delta_{i j}\right]^{2} .
$$

Kruskal $[28,26]$ proposed minimizing $\sigma(X)$ by an ad hoc gradient method in which the step length is determined by the angle between the present and preceding gradients. Guttman [21] observed that the stationary equation $\nabla \sigma(X)=0$ can be written as $X=C(X) X$, where the matrix-valued function $C$ depends on the dissimilarity matrix $\Delta$, and suggested that the sequence defined by the Guttman transform $X^{k+1}=C\left(X^{k}\right) X^{k}$ should converge to a stationary point of $\sigma$. This turned out to be essentially correct, and all of the algorithms for which convergence has been demonstrated are based on this idea.

The first rigorous analysis of convergence was supplied by de Leeuw [7] and elaborated upon by de Leeuw and Heiser [10] and de Leeuw [8]. Despite the fact that $\sigma$ is not everywhere differentiable (because the square root function is not differentiable at the origin, $\sigma$ is not differentiable at $X$ if some $d_{i j}(X)=0$, i.e. if some points in the configuration coalesce), the Guttman sequence is globally convergent to a connected set of local minimizers. In fact, de Leeuw (1984) [9] proved that $\sigma(X)$ is differentiable at all local minimizers, so that differentiability can be assumed for local convergence analysis. (Note that a consequence of this fact is that points cannot coalesce in optimal STRESS configurations.) The first such analysis was undertaken by de Leeuw [8], who concluded that "in almost all cases convergence is linear, with a convergence [constant] close to unity." (p. 163). This analysis explains the empirically observed fact that convergence of MDS algorithms is usually very slow.

To establish convergence, de Leeuw [8] assumed that the configurations were centered at the origin. (This can be ensured by starting with a centered configuration, since this property is preserved by the Guttman transform.) This assumption removes some, but not all, of the isometric indeterminacy that characterizes Problem (3). De Leeuw subsequently observed that

"The discussion in the previous sections shows that at least part of the difficulty with proving actual convergence of our iterations comes from the rotational indeterminacy of multidimensional scaling. ... If we eliminate rotational indeterminacy, then we eliminate these difficulties." ( $p$. $175)$.

If rotational indeterminacy is eliminated (de Leeuw suggested rotating to principal components), then local minimizers may be isolated and de Leeuw obtained the rate at which the Guttman sequence converges to an isolated local minimizer.

Actually, there were other reasons to anticipate the linear convergence of the Guttman sequence. If differentiability of $\sigma(X)$ is assumed, then it is well-known that the sequence can be written as the iterates of a weighted gradient algorithm, $X^{k+1}=X^{k}-(1 / 2) V^{+} \nabla \sigma\left(X^{k}\right)$, for a certain fixed matrix $V^{+}$. In contrast, the method of steepest descent is a gradient algorithm that produces iterates of the form $X^{k+1}=X^{k}-$ $\alpha_{k} \nabla \sigma\left(X^{k}\right)$, where $\alpha_{k}$ is a (positive) real number. It is generally understood that gradient algorithms, which do not exploit information about second derivative behavior, typically exhibit linear rates of convergence.

Despite the slow convergence of gradient methods, their use has been strongly emphasized in the MDS literature. For example, Kruskal [26] distinguished between unconstrained optimization methods whose memory requirements are linear (Class 1, e.g. gradient methods) and more than linear (Class 2, e.g. Newton's method) in the number of variables, and commented:

"Although Classes 1 and 2 have both been used in this field, Class 1 has been used much more often. In addition to the high cost of memory during computing, this may be due to the fact that high accuracy solutions are almost never needed in this field due to the substantial random error which we typically find in the data. Since the solution is meaningful only up to a certain level due to random error in the input, there is no need to obtain a solution which is accurate to a much higher level. Hence the higher speed of convergence for Class 2 methods does not have so great an attraction." (p. 315). 
A more modern assessment of these issues is long overdue. First, both computers and computational mathematics have advanced enormously in the last seventeen years. Second, the problems of molecular conformation are very different from the problems of psychology. The number of objects is typically much greater (a protein molecule may contain thousands of atoms), and the dissimilarities are typically much more accurate. Third, the good semi-local properties of Newton's method when applied to mildly nonlinear objective functions (fast linear convergence in large neighborhoods of solutions) imply that there can be considerable gains from using Newton's method even when high accuracy is not required.

One fairly conservative possibility for accelerating the convergence of the Guttman sequence is to modify the step choice in the corresponding gradient algorithm. This is precisely the motivation for Kruskal's [28, 26] angle-dependent gradient method. De Leeuw and Heiser [10] presented a simple device that "approximately halves the number of iterations required to obtain a given precision, at no extra cost." (p. 513). De Leeuw (1988) [8] suggested that this improvement was what could generally be expected from such modifications, and concluded that "one should always study the second derivatives of the loss function at the stopping point of the algorithm." (p. 179).

In Sections 4 and 5, we will argue that it is desirable to study the second derivative at each iteration of the algorithm. The present aversion to so doing appears to be largely due to the perception that it is a prohibitively expensive course of action. Thus, recent advances have attempted compromises. One such compromise is the gradient method of Barzilai and Borwein [1], which determines step length using a Rayleigh quotient that approximates an eigenvalue of the Hessian matrix. Local convergence properties of this method were established by Raydan [36].

Glunt, Hayden, and Raydan [17] applied the Barzilai and Borwein method to the problem of minimizing $\sigma(X)$. The authors assumed that the starting configuration is centered, in which case all subsequent configurations are necessarily centered; however, they did not attempt to remove rotational indeterminacy. They refer to this new algorithm as the spectral gradient method, and they found that its use decreased the cpu time required by de Leeuw's [8] implementation of the Guttman sequence (which they called the majorization algorithm) by a factor of 10-20. Even greater acceleration is obtained with use of a preconditioner (Glunt, Hayden, and Raydan [18]).

In its present form, the spectral gradient algorithm has faster local convergence than the majorization algorithm, but it sacrifices global convergence. The lack of global convergence requires construction of a good starting configuration; however, as Glunt, Hayden, and Raydan [17] point out, even globally convergent methods require good starting configurations to obtain "good" local minimizers. Because their technique for constructing a starting configuration for the spectral gradient method involves solving a metric SSTRESS problem, and is actually the same technique employed by Glunt, Hayden, and Liu [16], we defer discussing it until Section 3.2. Virtually all of the methods that have been proposed in the MDS liter ature for constructing starting configurations involve solving an MDS problem that is presumed to be easier than whichever one is actually under investigation.

\subsection{SSTRESS}

The SSTRESS criterion is the objective function in Problem (3) obtained by setting $r=1$. Computationally, it is considerably more manageable than the STRESS criterion (in particular, it is everywhere smooth), and was in fact proposed for this reason. However, there is no analogue of the Guttman transform for SSTRESS. For this reason, the methods proposed for the metric SSTRESS problem have differed from those proposed for the metric STRESS problem.

For the special case of dimension $p=n$, an extensive analysis of the metric SSTRESS problem was provided by Glunt, Hayden, Hong, and Wells [15]. In this case, the dimension of the solution is not constrained, the constraint set $\mathcal{D}_{n}(p)$ is convex, and a global minimizer of SSTRESS in the unweighted case can be obtained using the authors' Modified Alternating Projection (MAP) algorithm. Of course, the solution is typically of very high dimension. Because most applications require a low-dimensional configuration, e.g. $p=2,3$, the configuration constructed by the MAP algorithm is not of much interest per se.

Until recently, the best algorithm for the case of dimension $p<n-1$ was the one proposed by Browne [5]. To understand the basis for this algorithm, it is necessary to briefly digress and consider the classical metric scaling technique of Torgerson [42].

Given a dissimilarity matrix $\Delta$, let $\Delta * \Delta$ denote the matrix whose elements are the squared dissimilarities. 
Let $\tau: A \rightarrow B$ denote the linear operator defined by

$$
b_{i j}=-\frac{1}{2}\left(a_{i j}-\bar{a}_{i .}-\bar{a}_{\cdot j}+\bar{a}_{. .}\right),
$$

often called "double centering" by psychometricians. Then a well-known embedding theorem from classical distance geometry states that an $n \times p$ configuration matrix $X$ satisfies $D(X)=\Delta$ if and only if $\tau(\Delta * \Delta)=$ $X X^{T}$. Thus, the cone of distance matrices is parametrized by the cone of positive semidefinite matrices of rank $\leq p$, and a configuration matrix can be obtained from a positive semidefinite matrix by factorization.

In case $\Delta$ is not a distance matrix, Torgerson [42] proposed solving the optimization problem

$$
\begin{array}{ll}
\text { minimize } & \operatorname{tr}\left[(B-\tau(\Delta * \Delta))^{2}\right] \\
\text { subject to } & B \in \Omega_{n}(p),
\end{array}
$$

where $\operatorname{tr}(\cdot)$ denotes the trace operator. Equivalently, the objective function in this problem is the square of the Frobenius norm (the $L^{2}$ norm on $R^{n \times n}$ ) of $B-\tau(\Delta * \Delta)$. This function was subsequently dubbed STRAIN, making Problem (4) the metric STRAIN problem. The metric STRAIN problem has the very attractive feature that one can compute an explicit global solution $B^{*}$. Let

$$
\lambda_{1} \geq \cdots \geq \lambda_{n}
$$

denote the eigenvalues of $\tau(\Delta * \Delta)$, let $\Lambda=\operatorname{diag}\left(\lambda_{1}, \ldots, \lambda_{n}\right)$, and let $U \Lambda U^{T}$ be the spectral decomposition of $\tau(\Delta * \Delta)$. Define $\bar{\Lambda}$ by $\bar{\lambda}_{i}=\max \left(\lambda_{i}, 0\right)$ for $i=1, \ldots, p$ and $\bar{\lambda}_{i}=0$ for $i=p+1, \ldots, n$. Then $B^{*}=U \bar{\Lambda} U^{T}$. For further details about the metric STRAIN problem (and the embedding result on which it is based), see the review article by Trosset [44].

We now return to Browne's [5] algorithm for the metric SSTRESS problem. Given a dissimilarity matrix $\Delta$, the SSTRESS criterion in the unweighted case is

$$
f(X)=\operatorname{tr}\left[(\Delta * \Delta-D(X) * D(X))^{2}\right] .
$$

To eliminate isometric indeterminacy, Browne introduced a "duplicate" configuration matrix $Y$ and penalized $X$ for departures from $Y$. The discrepancy between $X$ and $Y$ was measured using the STRAIN criterion. Then, for a constant "deceleration" scalar $\alpha \in(0,1]$, Browne proposed minimizing the objective function

$$
f^{*}(X, Y)=f(X)+4 \frac{1-\alpha}{\alpha} \operatorname{tr}\left[\left(Y Y^{T}-X X^{T}\right)^{2}\right] .
$$

Thus, Browne added free variables to the metric SSTRESS problem in an attempt to make it easier to solve.

Browne suggested choosing the initial $X$ configuration to be the solution to the metric STRAIN problem. His algorithm for minimizing (5) involves alternately minimizing $f^{*}\left(X_{n}, Y\right)$ for $X_{n}$ fixed to obtain $Y_{n}=X_{n}$, then minimizing $f^{*}\left(X, Y_{n}\right)$ for $Y_{n}$ fixed to obtain $X_{n+1}$. This is the method of variable alternation for reducible nonlinear programming; in the context of MDS, it is usually called the method of alternating least squares (ALS).

To accomplish the nontrivial minimization subproblem in the ALS formulation, Browne's algorithm uses Newton's method. Because this fact has been emphasized in the MDS literature, we stress that Browne's algorithm is not equivalent to applying Newton's method to the (unweighted) metric SSTRESS problem. In fact, when ALS converges, it usually does so at only a linear rate.

The superiority of Browne's algorithm over its predecessors was described by Glunt, Hayden, and Liu [16], who stated:

"A number of algorithms have been proposed for the solution of the [metric SSTRESS] problem by researchers in multidimensional scaling. An algorithm due to de Leeuw and Takane was modified by Browne (1987) by adding a Newton Raphson step (henceforth called the NR method) and resulted in the best algorithm known to us for finding a local minimum solution of the [metric SSTRESS] problem. [NR] either finds the global minimum (about $90 \%$ of the time in our examples) or a local minimum with objective function near the global minimum. Furthermore, NR is orders of magnitude faster than competing algorithms and hence NR is our current yardstick for measuring success." (p. 770). 
Measured by the Browne yardstick, the algorithm subsequently proposed by Glunt, Hayden, and Liu [16] is extremely impressive. In its first phase, the MAP algorithm is used to (approximately) solve the metric SSTRESS problem with $p=n$. (Glunt, Hayden, Hong, and Wells [15] had found that MAP was approximately four times faster than Browne's algorithm for solving this problem.) The MAP solution is then used to produce a starting point for the second phase, in which a penalty term is added to the objective function (to remove translation invariance) and a local minimizer is obtained by use of "a standard (say quasi-Newton) unconstrained nonlinear optimization routine, with analytic gradient computed by ...." (p. 788). Glunt, Hayden, and Liu found that this "two-phase" algorithm was approximately ten times faster than Browne's algorithm.

Let us make several observations about the algorithm of Glunt, Hayden, and Liu [16]. First, it is not at all clear that the expense of using the MAP algorithm in the first phase is justified. Let $\Delta^{0}$ denote the given dissimilarity matrix and let $D^{0} \in \mathcal{D}_{n}(p)$ denote the distance matrix obtained from the MAP algorithm. Then Glunt, Hayden, and Liu (and also Glunt, Hayden, and Raydan [17] for the metric STRESS problem; see Section 3.1) chose, as a starting configuration matrix $X^{0}$, a configuration that solves the metric STRAIN problem with $\Delta=D^{0}$. We see no obvious reason why this should be superior to Browne's [5] considerably less expensive choice of a configuration that solves the metric STRAIN problem with $\Delta=\Delta^{0}$, the original dissimilarity matrix.

Second, although Glunt, Hayden, and Liu [16] were quite careful to remove translation invariance, their penalty function does not remove rotational invariance. This was overlooked by the authors, who mistakenly believed that the Hessian matrix of their objective function is necessarily positive definite at local minimizers. Of course, it is not difficult to modify the penalty function so that rotational invariance is also removed. This was done, for example, by Tarazaga and Trosset [41], who used the parametrization $B=X X^{T}$ to study optimization problems defined on the set of symmetric positive semidefinite matrices of rank $\leq p$. The difficulty with this entire approach, however, is that it demands a great deal of the penalty function. In practice, devices of this sort tend to lead to a deterioration of the local behavior of the algorithm.

Finally, although Glunt, Hayden, and Liu [16] emphasized their use of the analytic gradient vector, they declined to use the analytic Hessian matrix. In fact, they opined that "The formula for the Hessian appears too complicated to be computationally helpful in the general case." (p. 779). This was also the opinion of Glunt, Hayden, and Raydan [17] with regard to the metric STRESS problem. As we have already remarked, however, extensive numerical experimentation is usually required to determine whether or not it pays to compute second derivatives. We now consider an algorithm that, it turns out, makes extremely efficient use of such information.

\section{A New Algorithm}

We begin by writing Problem (3) as a standard nonlinear least-squares problem. To do this, let $m=$ $n(n-1) / 2$ denote the number of interpoint dissimilarities (or distances), define $G_{r}: R^{n \times p} \rightarrow R^{m}$ to have component functions of the form

$$
\left(\sum_{k=1}^{p}\left(x_{i k}-x_{j k}\right)^{2}\right)^{r}-\left(\delta_{i j}^{2}\right)^{r}
$$

for $i<j$, and let $W \in R^{m \times m}$ be the diagonal matrix whose diagonal elements are the weights $w_{i j}$ associated with the corresponding interpoint dissimilarities $\delta_{i j}$. Then Problem (3) can be written as the nonlinear least-squares problem

$$
\text { minimize } f_{r}(X)=\frac{1}{2} G_{r}(X)^{T} W G_{r}(X) .
$$

In Section 2, we remarked that the objective function $f_{r}(X)$ is invariant under isometric transformations of the configuration matrix $X$. For reasons discussed there, we want to remove the unnecessary degrees of freedom that result from translational and rotational invariance. As illustrated by each of the methods reviewed in Section 3, there is a long tradition in MDS of removing translational invariance by centering the configuration at the origin. Rotational invariance has variously been removed by expensive procedures such as principal component analysis or simply ignored. We propose to abandon these traditions and make use of an elementary device that has so far been neglected by MDS researchers. 
In an early article concerned with finding molecular configurations that minimize the Lennard-Jones potential energy function, Hoare and Pal [25] described an elementary way of removing translational and rotational invariance in $R^{3}$. One simply constrains one point in the configuration to lie at the origin, specifies two coordinate axes, constrains a second point to lie along the first specified axis, and constrains a third point to lie in the plane determined by the two axes. Thus, for $X \in R^{n \times 3}$, we parametrized Problem (7) by setting

$$
X=\left[\begin{array}{lll}
0 & 0 & 0 \\
x_{1} & 0 & 0 \\
x_{2} & x_{3} & 0 \\
x_{4} & x_{5} & x_{6} \\
\cdot & \cdot & \cdot \\
\cdot & \cdot & \cdot \\
\cdot & \cdot & \cdot
\end{array}\right]
$$

Unless the selected points are collinear, this parametrization removes the isometric invariance of $f_{r}(X)$. In general, one selects $p$ points, fixing $p-k+1$ coordinates of point $k$, for $k=1, \ldots, p$. So long as the selected points are not contained in a subspace of dimension $p-2$, this parametrization removes the isometric invariance of $f_{r}(X)$.

Most of our numerical experiments have been performed for $X \in R^{n \times 3}$. In these experiments, we have found that collinearity of the selected points is virtually never encountered. In theory, of course, it may be that the three selected points are collinear in the minimizing configuration. One way of dealing with this possibility is to reparametrize, using a different triple of points, whenever the original triple is nearly collinear. If one can afford the expense of computing the metric STRAIN solution, then one can examine it to discover a triple that is almost certainly not collinear. (This also allows one to determine whether or not the dissimilarity matrix actually is a distance matrix of dimension less than $p$, e.g. all points collinear.) For the remainder of this paper, we assume that the points have been labelled in such a way that we are fixing the indicated coordinates of the first $p$ points.

The parametrization defined by (8) appears to be quite standard in the literature on minimizing the energy of molecular configurations. For example, Northby [34] used it quite casually to preclude translation or rotation of the configuration. This parametrization is rarely used in MDS. (The only example of which we are aware is by Groenen [20], who exploited it for global optimization of the metric STRESS problem by a multi-level single-linkage clustering algorithm.) One possible explanation of this fact is that MDS researchers may have been unduly influenced by Torgerson's [42] formulation of the metric STRAIN problem. Young's and Householder's [46] original solution of the embedding problem (the case in which the dissimilarity matrix actually is a distance matrix) placed the $n$th point of the configuration at the origin. Torgerson observed that, with "fallible data," different solutions are obtained according to which point is labelled the $n$ th. As an antidote, he introduced the double centering operator that we have denoted by $\tau$, which leads to configurations that are centered at the origin. Thus, for the metric STRAIN problem, configurations are centered in order to specify a solution that does not depend on the indexing of the points. However, it is quite clear that solutions to Problems (1), (3), and (7) do not depend on the indexing of the points. Hence, there is no substantive reason to require that solutions to the metric STRESS or SSTRESS problems be centered at the origin. Of course, if a centered solution is desired for aesthetic reasons, then one can always translate a noncentered solution after it has been obtained.

The parametrization defined by (8) reduces the number of free variables in Problem (7), from $n p$ to $N=$ $n p-p(p+1) / 2$. Thus, the simplified problem has objective function $f_{r}: R^{N}-R$, with $G_{r}: R^{N} \rightarrow R^{m}$ again having component functions defined by expression (6). The simple structure of $f_{r}$ makes it a straightforward matter to derive analytic expressions for $\nabla f_{r}$ and $\nabla^{2} f_{r}$. These expressions are not expensive to evaluate; in fact, $f_{r}, \nabla f_{r}$, and $\nabla^{2} f_{r}$ can be computed in $O(N)$ floating point operations. Using this analytic information may result in algorithms with smaller truncation errors and better stability properties - see Boggs and Dennis [3] for an error analysis.

Because Problem (7) is a nonlinear least-squares problem, it can be solved reasonably efficiently by applying any good general nonlinear least-squares algorithm. Such algorithms have been developed by Moré, Garbow, and Hillstrom [33], by Dennis, Gay, and Welsch [12], and by Boggs, Byrd, Donaldson, and Schnabel [2]. These algorithms, however, incorporate conservative precautions and very delicate globalization strategies designed for highly nonlinear problems. In contrast, the nonlinearity of the residual functions in 
Problem (7) is very mild. When the above algorithms are applied to mildly nonlinear problems, they tend to require more computation and result in longer run times than are necessary. Therefore, it makes sense to develop a more specialized algorithm for Problem (7).

It is important to appreciate that the Hessian matrix $\nabla^{2} f_{r}(X)$ is completely dense. In many applications, it is considerably less expensive to compute an alternative matrix, $\nabla G_{r}(X) \cdot \nabla G_{r}(X)^{T}$. Using this alternative matrix leads to the Gauss-Newton algorithms, which are particularly effective on so-called "small residual" problems. See Chapter 10 of Dennis and Schnabel [13] for a discussion of the well-known Levenberg-Marquardt version of this algorithm.

In numerical analysis, a small residual problem is one for which the value of the objective function at the solution is small compared to a typical value of the objective function. For example, $f_{r}\left(X^{*}\right)<<f_{r}\left(X^{0}\right)$ would be suggestive of a small residual problem. In this relative sense, Problem ( 7$)$ is typically a small residual problem. However, the availability and density of the Hessian matrix render negligible the relative savings from using the matrix $\nabla G_{r}(X) \cdot \nabla G_{r}(X)^{T}$ instead of the complete Hessian matrix $\nabla^{2} f_{r}(X)$. In fact, for very large problems, $\nabla G_{r}(X) \in R^{N \times m}$ is considerably more difficult than $\nabla^{2} f_{r}(X) \in R^{N \times N}$ to store and to use in forming matrix-vector products. In this way, Problem (7) differs from most small residual nonlinear least-squares problems.

All of the above considerations combine to suggest the viability of simply applying Newton's method to Problem (7). The specific algorithm that we implemented can be summarized as follows, where $G_{r}^{i}$ : $R^{N} \rightarrow R$ denotes the $i$ th component function of $f_{r}$ and $I$ denotes the $N \times N$ identity matrix. To facilitate implementation, we indicate relevant sections of Dennis and Schnabel [13]. Their Appendix A provides pseudocode for these modules.

\section{Globalized Newton's Method Algorithm}

1. Construct an initial configuration $X^{0}$.

2. Specify the convergence tolerances and a radius $\rho^{0}>0$ for the initial model trust region. Set $k=0$.

3. Compute the gradient $\nabla f_{r}\left(X^{k}\right)$ and the Hessian

$$
H^{k}=\sum_{i=1}^{m}\left[\nabla G_{r}^{i}\left(X^{k}\right) \cdot \nabla G_{r}^{i}\left(X^{k}\right)^{T}+G_{r}^{i}\left(X^{k}\right) \cdot \nabla^{2} G_{r}^{i}\left(X^{k}\right)\right]
$$

4. Determine the step direction $s^{k}$ by minimizing the local quadratic model for $f_{r}$ in the model trust region, i.e. solve the subproblem

$$
\begin{array}{ll}
\text { minimize } & f_{r}\left(X^{k}\right)+\nabla f\left(X^{k}\right)^{T} s+s^{T} H^{k} s / 2 \\
\text { subject to } & \|s\|_{2} \leq \rho^{k} .
\end{array}
$$

See Dennis and Schnabel [13], Section 6.4. Because there is no finite method for exactly solving this subproblem, we settle for an approximate solution. Our preference is for the hook-step solution of Hebden [23] and Moré [32]. See Dennis and Schnabel [13], Section 6.4.1 and Algorithms A6.4.1 and A6.4.2.

5. Determine the step length $\alpha^{k}$ by performing a backtracking line search on $f_{r}$. (It is somewhat nontraditional to backtrack from a trust-region step, but extensive numerical experimentation revealed that doing so slightly improves the overall performance of the algorithm on these problems.) See Dennis and Schnabel [13], Section 6.3.2 and Algorithm A6.3.1.

6. Set $X^{k+1}-X^{k}+\alpha^{k} s^{k}$.

7. Check convergence. See Dennis and Schnabel [13], Section 7.2 and Algorithm A7.2.1.

8. Update the radius of the model trust region, i.e. compute $\rho^{k+1}$. See Dennis and Schnabel [13], Section 6.4.3 and Algorithm A6.4.5.

9 . Set $k-k+1$ and go to Step 3 . 
We investigated two strategies for constructing the initial configuration $X^{0}$. These strategies represent two possible compromises in the unavoidable tradeoff between the quality of $f_{r}\left(X^{0}\right)$ and the expense of computing $X^{0}$. Historically, MDS researchers have eschewed inexpensive initial configurations and have attempted to obtain initial configurations located near desired solutions. The primary reason for this is concern about local minimizers - it is certainly plausible that better initial configurations will allow the algorithm to find better local minimizers.

In this spirit, our first strategy for constructing an initial configuration was to compute (a translation/rotation of) the metric STRAIN solution. As described in Section 3.2, this is precisely how Browne [5] constructed initial configurations and is very similar to the strategy employed by Glunt, Hayden, and Liu [16] and Glunt, Hayden, and Raydan [17]. This construction requires computing the spectral decomposition of the symmetric $n \times n$ matrix $\tau(\Delta * \Delta)$. Although modern methods for computing the spectral decomposition are very efficient (we employed a $k$-step Arnoldi method with implicit filtering; see Sorensen [39] for details), the fact that the matrix $\tau(\Delta * \Delta)$ is extremely dense means that implementing this strategy may still be fairly expensive when $n$ is large, as is often the case for molecular conformation problems.

In case computing resources are limited, we also investigated a second, less expensive strategy for constructing an initial configuration. This construction proceeds sequentially, beginning with the placement of the first point at the origin. In general, the $j$ th point is placed at a location determined by the preceding $p$ points. Specifically, given $x_{j-p}, \ldots, x_{j-1}$, the coordinates of $x_{j}$ are determined by solving the elementary least-squares problem

$$
\operatorname{minimize} \quad \sum_{i=j-p}^{j-1} w_{i j}\left[\left(\sum_{k=1}^{p}\left(x_{i k}-x_{j k}\right)^{2}\right)^{r}-\left(\delta_{i j}^{2}\right)^{r}\right]^{2} .
$$

Notice the similarity of Problems (9) and (3). Solving Problem (9) optimizes the location of $x_{j}$ with respect to $x_{i}(i=j-p, \ldots, j-1)$, either approximating the $p$ dissimilarites $\delta_{i j}$ with the $p$ distances $d_{i j}$ (the STRESS criterion, for $r=1 / 2$ ) or approximating the $p$ squared dissimilarites $\delta_{i j}^{2}$ with the $p$ squared distances $d_{i j}^{2}$ (the SSTRESS criterion, for $r=1$ ). This construction turns out to be considerably less expensive than computing the metric STRAIN solution, and our experience suggests that the algorithm typically converges to the same local minimizer from either initial configuration.

When points in a configuration coalesce, i.e. when the algorithm steps to a configuration matrix $X^{k}$ with two or more identical rows, the performance of the algorithm deteriorates and numerical difficulties are sometimes encountered. Naturally, we would prefer to avoid such configurations. For the STRESS problem, de Leeuw [9] has shown that points cannot coalesce at local minimizers, so there is no reason to ever consider such configurations. (In contrast, points can coalesce at local minimizers of the SSTRESS problem, and also at solutions of the STRAIN problem. This is one argument that can be advanced for preferring the STRESS criterion.) We found that, in practice, configurations with coalescing points are rarely encountered if all points in the initial configuration are distinct. For this reason, we actually implemented a slightly modified version of our second strategy for choosing an initial configuration. If solving Problem (9) placed $x_{j}$ too close to any previous points, then the location of $x_{j}$ was perturbed. We required that no interpoint distance in the initial configuration be smaller than the smallest (strictly positive) dissimilarity, i.e.

$$
\min _{i j} d_{i j}\left(X^{0}\right) \geq \min _{i j} \delta_{i j}
$$

This condition was easy to enforce, and in practice it effectively inhibited the coalescence of points in subsequent configurations $X^{k}$.

We experimented with several implementations of globalization strategies. Both of the standard trustregion implementations performed extremely well. Extensive numerical experimentation suggested that the hook-step implementation of Hebden [23] and More [32] slightly outperformed the double dogleg step implementation of Powell [35]. When a line-search method was used instead of a trust-region method, the overall performance of the algorithm deteriorated. For this reason, our algorithm uses the hook-step implementation of the trust-region method to determine a step direction. Notice, however, that we then backtrack from the hook step. As we have already remarked, backtracking from a trust-region step is somewhat nontraditional, but extensive numerical experimentation suggested that it marginally improved the overall performance of the algorithm.

Following Section 7.2 of Dennis and Schnabel [13], our convergence criterion combined three different conditions, developed to answer the following heuristic questions: 
1. "Have we solved the problem?"

2. "Have we ground to a halt, either because the algorithm has converged or simply because it has stalled?"

3. "Have we exhausted our resources?"

To ascertain if the problem has been solved, we check to see if we have found a stationary configuration, i.e. a configuration at which the gradient of the objective function is sufficiently close to zero. In case the problem is badly scaled, we employ a relative measure of magnitude. Let typ $x_{i}$ denote the user's estimates of typical magnitudes of the coordinates of $X$, and let typ $f$ denote the user's estimate of a typical magnitude of $f_{r}(X)$. Then the condition suggested by Dennis and Schnabel [13] is

$$
\max _{1 \leq i \leq N} \frac{\left|\left[\nabla f_{r}\left(X^{k}\right)\right]_{i}\right| \max \left(\left|\left[X^{k}\right]_{i}\right|, \operatorname{typ} x_{i}\right)}{\max \left(\left|f_{r}\left(X^{k}\right)\right|, \operatorname{typ} f\right)} \leq \epsilon_{1} .
$$

To ascertain if the algorithm has ground to a halt, we check to see if the size of the step is sufficiently close to zero. Again, we employ a relative measure of magnitude, viz.

$$
\max _{1 \leq i \leq N} \frac{\left|\left[s^{k}\right]_{i}\right|}{\max \left(\left|\left[X^{k}\right]_{i}\right|, \operatorname{typ} x_{i}\right)} \leq \epsilon_{2}
$$

Finally, we limit resources by limiting the number of iterations. The algorithm continues until either condition (10) or condition (11) is satisfied, or until the maximum number of iterations is reached.

\section{$5 \quad$ Numerical Experiments}

The algorithm presented in Section 4 was tested on several large, unweighted metric STRESS problems. For small problems, algorithmic efficiency is obviously less important. Currently, the most important source of large metric MDS problems is computational chemistry. For this reason, our test problems were designed to approximate molecular structure and configurations were constructed in $p=3$ dimensions.

There were several reasons for our focus on the STRESS criterion. First, STRESS problems appear to be more difficult than SSTRESS problems. Second, it is our impression that SSTRESS has declined in popularity in recent years. Third, STRESS seems particularly suitable for molecular conformation because, unlike SSTRESS, points cannot coalesce in optimal STRESS configurations. Fourth, unlike SSTRESS, there is an established computer program, SMACOF-I [24], for minimizing the STRESS criterion. The SMACOFI program, which is an implementation of the majorization method discussed in Section 3.1, provides a "gold standard" to which our algorithm can be compared. It should be noted that SMACOF-I is highly regarded in the literature. For example, McFarlane and Young [31] recently stated that "In the context of an interactive and dynamic graphics system, the method of choice is SMACOF-I ...; it is the fastest algorithm that optimizes the [STRESS criterion]." (p. 26).

We began by selecting three molecules: Cranbin $(n=394$ atoms), Deoxyribonucleic acid $(n=566$ atoms), and Glycopeptide antibiotic ( $n=122$ atoms). Configuration coordinates in $R^{3}$ for these molecules are available from the Brookhaven Protein Bank. From these coordinates, we computed the Euclidean interatomic distances, $\bar{d}_{i j}$. These distances were then perturbed to obtain dissimilarities.

For each molecule, we constructed three dissimilarity matrices, each having a different magnitude of error. The true interatomic distances $\bar{d}_{i j}$ were multiplied by errors drawn from a lognormal distribution, an error model proposed by Wagenaar and Padmos [45] that was recently employed by Groenen [20]. Specifically, for each $\bar{d}_{i j}$, we generated a pseudorandom number $z_{i j}$ from a standard normal distribution. We then set $\sigma=g(\log 10) / 1.95996$, for $g=1,2,3$, and $\delta_{i j}=\bar{d}_{i j} \exp \left(\sigma z_{i j}\right)$.

For each of the nine dissimilarity matrices that we obtained, we attempted to solve the metric STRESS problem in $p=3$ dimensions using three different algorithms: our implementation of Newton's method, described in Section 4; and each of the two updating schemes available in SMACOF-I, "Guttman transforms" and "relaxed updates." Heiser and de Leeuw [24] argued that the latter scheme squares the convergence constant, thereby halving the number of iterations required for convergence by the Guttman sequence. 
For each of the nine dissimilarity matrices, we also considered three strategies for choosing an initial configuration: the metric STRAIN solution, which happens to be the default initial configuration computed by SMACOF-I; the less expensive configuration described in Section 4; and five randomly generated configurations. In order for the randomly generated configurations to be meaningful, it is important to ensure that they are reasonably scaled.

Given a dissimilarity matrix $\Delta$, a fairly natural way to generate a random initial $n \times p$ configuration matrix $Y$ is to do the following. Let $m=n(n-1) / 2$, let $S=\sum_{i<j} \delta_{i j}$, and let $\sigma^{2}=S /(2 p m)$. For each configuration coordinate, generate a pseudorandom number $z_{i j}$ from a standard normal distribution and take $y_{i j}=\sigma z_{i j}$. It is easily verified that this procedure produces configurations for which the expected squared interpoint distance equals the average squared dissimilarity in $\Delta$.

To perform the indicated numerical experiments, we had to overcome several technical obstacles. First, it was necessary to slightly modify SMACOF-I so that sufficient memory was allocated to solve the very large problems that we considered. Despite the fact that SMACOF-I does not require second derivatives and our algorithm does, the size of the problems that we were able to consider on our platform (Sun SparcStation 10) was smaller for SMACOF-I than for our algorithm.

A more serious difficulty is the convergence criterion used by SMACOF-I, which stops when an iteration fails to decrease the value of the STRESS function by at least $\epsilon_{3}$. (The value of $\epsilon_{3}$ can be specified by the user; the SMACOF-I default is $\epsilon_{3}=10^{-5}$.) This criterion may stop the algorithm prematurely, as failure to take a step that sufficiently decreases the value of the objective function does not necessarily mean that one is near a local minimizer. We addressed this difficulty by using each solution found by SMACOF-I as an initial configuration for our algorithm, thereby establishing if further decrease was possible.

Finally, it should be noted that these experiments required a certain amount of recoordinatization. Configurations must be centered if they are to be used as initial configurations for SMACOF-I, whereas our algorithm uses a different parametrization.

Because SMACOF-I was written in single precision, all of our experiments were performed using 32-bit IEEE floating point arithmetic. In consequence, the STRESS values that we obtained are presumed to have only 3-4 accurate digits. Naturally, our algorithm produces more accurate solutions when it is run in double precision. We specified typical values to be $\operatorname{typ} x_{i}=0.1$ and $\operatorname{typ} f=f_{r}\left(X^{0}\right) / 10$, and the radius of the initial model trust region to be

$$
\rho^{0}=\left\|\operatorname{diag}\left(\operatorname{typ} x_{i}\right) \nabla f_{r}\left(X^{0}\right)\right\|_{2}=\left\|\nabla f_{r}\left(X^{0}\right)\right\|_{2} / 10 .
$$

Our algorithm stopped if either condition (10) or condition (11) was satisfied. We used toler ances of $\epsilon_{1}=10^{-6}$ and $\epsilon_{2}=10^{-8}$. Typically, the algorithm stopped because (10) was satisfied. The stopping criterion tolerance for SMACOF-I was $\epsilon_{3}=2 \times 10^{-6}$. Each run of each algorithm was permitted a maximum of 250 iterations.

The results of our experiments are tabled in the Appendix. From these tables, several patterns emerge. First, the hope that SMACOF-I's relaxed updating scheme requires approximately half as many iterations as Guttman transforms appears to be overly optimistic. Using the STRAIN solution as the initial configuration (the default for SMACOF-I), the ratio of the number of iterations for relaxed updates to the number of iterations by Guttman transforms ranged (over the nine dissimilarity matrices) from 0.5926 to 2.0541 , with a median of 0.7353 .

Next, it is quite interesting to note that the number of SMACOF-I iterations does not increase greatly with decreasing quality of the initial configuration. The median ratio of the number of iterations from the inexpensive initial configuration to the number of iterations from the STRAIN solution was actually less than unity ( 0.5833 for Guttman transforms, 0.6800 for relaxed updates). For random initial configurations, the corresponding median ratios were only 1.0556 for Guttman transforms and 1.1071 for relaxed updates. This phenomenon may be due to one or more of several possible causes. First, it may be a consequence of excellent global, but slow local, convergence properties of SMACOF-I. Second, it may be that SMACOF-I is finding different local minimizers from different initial configurations. Third, it may be that SMACOF-I is stopping prematurely. We do not attempt an exhaustive examination of these possibilities in this report. However, we will argue below that SMACOF-I does have a tendency to stop prematurely.

In contrast to SMACOF-I, the number of Newton iterations varies dramatically with the initial configuration. From the STRAIN solution, the number of iterations until convergence ranged (over the nine dissimilarity matrices) from 12 to 29 . The median ratio of the number of iterations from the inexpensive initial configuration to the number of iterations from the STRAIN solution was 2.5625 . From random initial configurations, the number of iterations until convergence was never less than 158 and the algorithm failed to 
converge in 250 iterations on several occasions. The median ratio of the smallest number of iterations from one of five random initial configuration to the number of iterations from the STRAIN solution was 7.6957. These results clearly illustrate the fast local convergence of Newton's method and the virtue of starting from a good initial configuration.

The most intriguing results have to do with the quality of the solutions obtained by the different algorithms from the different initial configurations. When solutions obtained by SMACOF-I were used as initial configurations for our algorithm, Newton's method always took additional steps and usually decreased the value of the objective function. From the SMACOF-I solutions obtained using Guttman transforms (relaxed updates) from the STRAIN solution, our algorithm took a median of 18 (18) additional steps and further decreased the STRESS value by a median of $0.77(0.45)$ percent. Considering the stringency of the tolerances in the convergence criteria, this an appreciable amount. When other SMACOF-I solutions were used, the numbers of additional steps and the relative decreases in STRESS were typically even greater.

For each dissimilarity matrix, starting SMACOF-I from different initial configurations typically produced different final STRESS values. In comparison, for each dissimilarity matrix our algorithm typically produced fairly homogenous final STRESS values regardless of the initial configuration. (Recall that the reported STRESS values cannot be presumed to have more than 3-4 accurate digits.) In particular, our algorithm typically recovered roughly the same final STRESS value when started from SMACOF-I solutions with rather different STRESS values. These results strongly suggest that SMACOF-I has a tendency to stop prematurely.

To some extent, SMACOF-I's tendency to stop prematurely can be counteracted by specifying an extremely stringent tolerance for its convergence criterion. We suspect, however, that the actual difficulty is more fundamental, as it is well known that the stopping criterion used by SMACOF-I (requiring each iteration to decrease the value of the objective function by at least the specified tolerance) has a general tendency to induce this behavior. Even more interesting is the question of whether the convergence criterion is entirely to blame or whether the algorithm itself is partially responsible. We believe that, because the STRESS function is extremely shallow (i.e. fairly large regions of configurations have very slowly varying STRESS values), any algorithm that fails to exploit second order information will experience difficulty actually locating a minimizer.

\section{Discussion}

Both the metric STRESS and SSTRESS problems are mildly nonlinear, but completely dense, least-squares problems for which local minimizers can be efficiently obtained by the methods of modern numerical optimization. These problems are well-suited to a straightforward application of Newton's method. Accordingly, we believe that the second order algorithm that we have presented represents a substantial improvement on the first order methods most commonly used in current practice. An additional, very appealing feature of this approach is that the same algorithm can be used with either the STRESS or the SSTRESS criterion.

Historically, MDS researchers have been reluctant to use second order methods because of their perception that memory is too expensive to warrant storing the Hessian matrix. This perception is challenged by our algorithm's ability to efficiently solve very large metric STRESS problems. In fact, as we have already noted, the size of the problem required to exhaust the memory available on our platform was greater for our second order algorithm than for the well-known first order algorithm SMACOF-I. Nevertheless, one can always pose a problem so large that it is impossible to store the Hessian matrix. For such problems, another advantage of our approach is that it is easily modified to exploit the limited memory quasi-Newton methods that have been developed for large-scale optimization. (See Gilbert and LeMaréchal [14] and Liu and Nocedal [30] for an introduction to these methods.) In preliminary testing, we have efficiently solved extremely large metric STRESS problems using a limited memory BFGS variant of our algorithm.

This paper has focussed exclusively on algorithms for finding solutions that correspond to fixed dissimilarity matrices. It is the fact that the dissimilarities are fixed that is the defining characteristic of metric MDS. Although metric MDS is a central mathematical problem of MDS, in most applications it does not represent the entire problem. For this reason, we conclude by briefly considering the importance of metric MDS and the potential role of the algorithm that we have presented.

In computational chemistry, MDS is sometimes used to construct molecular configurations from measure- 
ments of interatomic distances. Because a physical molecule actually does exist in $R^{3}$, dissimilarity matrices that lead to configurations with small objective function values are to be expected and preferred. Therefore, instead of simply solving the metric MDS problem defined by setting the dissimilarites equal to the measured interatomic distances, it is standard practice to begin by smoothing the data, obtaining a dissimilarity matrix that is more nearly a distance matrix. Nevertheless, and regardless of the exact procedure by which the dissimilarites are obtained, the solution of a metric MDS problem is an important component of the complete analysis.

Algorithms for solving metric MDS problems play a clearly defined role in Havel's [22] modularized DG-II package for the determination of protein structure from distance constraints obtained from nuclear magnetic resonance (NMR) spectroscopy. The majorization module of DG-II finds local minimizers of metric STRESS problems using de Leeuw's [8] majorization algorithm, described in Section 3.1. It would be a simple matter to replace this module with an implementation of the Newton method algorithm that we have proposed.

A slightly different approach to determining molecular structure is the data box algorithm of Glunt, Hayden, and Raydan [17], in which the dissimilarities are allowed to vary subject to bound constraints determined by the error structure of the measurement process. (For an algorithm that can be applied if the distances rather than the dissimilarities are bound-constrained, see Boggs, Tolle, and Kearsley [4].) The data box algorithm employs the method of alternating least squares (ALS), whereby one alternately optimizes the dissimilarity variables for a fixed configuration and the configuration coordinates for a fixed dissimilarity matrix. The latter subproblems are metric STRESS problems, which the authors solve using their spectral gradient algorithm. It would be a simple matter to substitute the Newton method algorithm for the spectral gradient algorithm.

Finally, as defined by Kruskal [27], nonmetric MDS allows the dissimilarities to vary subject to order constraints. As exemplified by the popular A LSCAL algorithm of Takane, Young, and de Leeuw [40], ALS is often used to solve nonmetric MDS problems. This means that, like the data box algorithm, many nonmetric MDS algorithms entail solving metric MDS subproblems. Again, it would be a simple matter to use the Newton method algorithm to solve these subproblems.

Thus, metric MDS problems appear in a variety of contexts. Many of these contexts involve large configurations and/or the successive solution of repeated metric MDS subproblems. Accordingly, one can reasonably anticipate that the very efficient Newton method algorithm for solving these problems will find a variety of applications.

\section{Acknowledgements}

We would like to thank the individuals who so generously contributed to our research. Mark Gockenbach took an interest in our work and made many helpful observations. Jan de Leeuw, Patrick Groenen, Tom Hayden and Marcos Raydan shared the results of their own studies of metric MDS problems. David Xi and especially Garry King kindly explained some of the relevant concerns of computational biochemistry and NMR spectroscopy, and also provided the molecular data sets that we used in our numerical experiments. Finally, we are especially grateful to Andrea Reiff, whose assistance in the development of our algorithm was invaluable.

\section{Appendix: Results of Numerical Experiments}

The following tables report numbers of iterations and final STRESS values for the numerical experiments described in Section 5. Each table corresponds to one of nine dissimilarity matrices. For each dissimilarity matrix, seven initial configurations were generated: the metric STRAIN solution, the inexpensive (Cheap) initial configuration described in Section 4, and five random initial configurations. Each row in each table corresponds to one initial configuration.

From each initial configuration, three algorithms were used to solve the metric STRESS problem: the algorithm proposed in Section 4 (Newton), SMACOF-I with Guttman transforms, and SMACOF-I with relaxed updates. For our algorithm, we report the number of iterations (Iter) and the final STRESS value. For example, consider the table for the Cranbin molecule with $g=1$. From the metric STRAIN solution, our algorithm converged in 13 iterations to a configuration with a STRESS value of 3356.61 . 
For the two SMACOF-I algorithms, we report the number of iterations (It-1) and the final STRESS value (Stress-1). For example, from the metric STRAIN solution for Cranbin with $g=1$, SMACOF-I with Guttman transforms converged in 27 iterations to a configuration with a STRESS value of 3463.58 and SMACOF-I with relaxed updates converged in 25 iterations to a configuration with a STRESS value of 3466.35. We also report the number of iterations (It-2) and the final STRESS value (Stress-2) obtained by starting our algorithm from the solution obtained by SMACOF-I. Thus, in the preceding example, from the solution obtained by SMACOF-I with Guttman transforms, our algorithm took 15 additional steps and decreased the STRESS value from 3463.58 to 3356.53 ; from the solution obtained by SMACOF-I with relaxed updates, our algorithm took 18 additional steps and decreased the STRESS value from 3466.35 to 3356.45 .

If an algorithm stopped because the maximum number of iterations (250) was reached, then the number of iterations is reported as 250 and no STRESS value is reported. To facilitate interpretation, the reported STRESS values are the computed STRESS values multiplied by $10^{4}$. Because single precision arithmetic was used, these values should be construed to have 3-4 accurate digits.

Cranbin, $\mathrm{g}=1$

\begin{tabular}{|l|rc|rrrr|rrrr|}
\hline & \multicolumn{2}{|c|}{ Newton } & \multicolumn{3}{|c|}{ SMACOF-I, Guttman transforms } & \multicolumn{4}{c|}{ SMACOF-I, Relaxed updates } \\
& Iter & Stress & It-1 & Stress-1 & It-2 & Stress-2 & It-1 & Stress-1 & It-2 & Stress-2 \\
\hline Strain & 13 & 3356.61 & 27 & 3463.58 & 15 & 3356.53 & 25 & 3466.35 & 18 & 3356.45 \\
\hline Cheap & 121 & 3356.59 & 41 & 3712.13 & 19 & 3356.44 & 40 & 3715.03 & 40 & 3356.62 \\
\hline Random & 219 & 3356.67 & 89 & 3915.29 & 159 & 3356.24 & 51 & 3723.99 & 226 & 3356.82 \\
\hline Random & 194 & 3356.62 & 56 & 3821.28 & 147 & 3356.80 & 49 & 3665.21 & 213 & 3357.36 \\
\hline Random & 250 & - & 45 & 3741.43 & 191 & 3356.03 & 36 & 3688.70 & 107 & 3356.87 \\
\hline Random & 183 & 3356.61 & 71 & 3970.98 & 212 & 3356.31 & 60 & 3731.75 & 186 & 3356.44 \\
\hline Random & 197 & 3356.62 & 31 & 3600.30 & 214 & 3356.49 & 29 & 3818.09 & 145 & 3356.37 \\
\hline
\end{tabular}

Cranbin, $\mathrm{g}=2$

\begin{tabular}{|l|rc|rrrr|rrrr|}
\hline & \multicolumn{2}{|c|}{ Newton } & \multicolumn{4}{|c|}{ SMACOF-I, Guttman transforms } & \multicolumn{4}{c|}{ SMACOF-I, Relaxed updates } \\
& Iter & Stress & It-1 & Stress-1 & It-2 & Stress-2 & It-1 & Stress-1 & It-2 & Stress-2 \\
\hline Strain & 12 & 3492.08 & 36 & 3545.62 & 18 & 3489.77 & 24 & 3546.67 & 16 & 3490.30 \\
\hline Cheap & 77 & 3492.34 & 21 & 3625.96 & 96 & 3492.52 & 21 & 3526.41 & 93 & 3490.76 \\
\hline Random & 175 & 3492.02 & 27 & 3670.84 & 126 & 3491.26 & 26 & 3506.19 & 145 & 3491.40 \\
\hline Random & 220 & 3492.21 & 67 & 3940.87 & 243 & 3492.79 & 41 & 3719.92 & 140 & 3491.09 \\
\hline Random & 235 & 3491.98 & 72 & 3593.05 & 118 & 3492.16 & 41 & 3618.18 & 109 & 3492.86 \\
\hline Random & 244 & 3492.06 & 38 & 3766.69 & 199 & 3490.69 & 37 & 3603.03 & 231 & 3489.61 \\
\hline Random & 219 & 3492.19 & 44 & 3697.08 & 145 & 3491.44 & 33 & 3564.30 & 218 & 3491.56 \\
\hline
\end{tabular}

Cranbin, $\mathrm{g}=3$

\begin{tabular}{|l|rc|rrrr|rrrr|}
\hline & \multicolumn{2}{|c|}{ Newton } & \multicolumn{3}{|c|}{ SMACOF-I, Guttman transforms } & \multicolumn{4}{c|}{ SMACOF-I, Relaxed updates } \\
& Iter & Stress & It-1 & Stress-1 & It-2 & Stress-2 & It-1 & Stress-1 & It-2 & Stress-2 \\
\hline Strain & 16 & 4191.81 & 81 & 4211.96 & 35 & 4191.53 & 48 & 4210.67 & 26 & 4191.58 \\
\hline Cheap & 41 & 4192.36 & 17 & 4303.55 & 195 & 4192.37 & 17 & 4333.25 & 232 & 4191.75 \\
\hline Random & 163 & 4191.99 & 49 & 4410.31 & 137 & 4191.84 & 41 & 4221.38 & 242 & 4191.94 \\
\hline Random & 223 & 4191.87 & 52 & 4349.89 & 216 & 4191.56 & 29 & 4221.12 & 99 & 4191.63 \\
\hline Random & 228 & 4191.69 & 54 & 4264.91 & 248 & 4192.78 & 31 & 4313.75 & 213 & 4192.09 \\
\hline Random & 250 & - & 24 & 4741.30 & 219 & 4192.16 & 22 & 4338.27 & 227 & 4191.73 \\
\hline Random & 250 & - & 43 & 4639.14 & 239 & 4191.27 & 41 & 4226.51 & 248 & 4191.99 \\
\hline
\end{tabular}


Deoxyribonucleic acid, $\mathrm{g}=1$

\begin{tabular}{|l|rc|rrrr|rrrr|}
\hline & \multicolumn{2}{|c|}{ Newton } & \multicolumn{4}{|c|}{ SMACOF-I, Guttman transforms } & \multicolumn{4}{c|}{ SMACOF-I, Relaxed updates } \\
& Iter & Stress & It-1 & Stress-1 & It-2 & Stress-2 & It-1 & Stress-1 & It-2 & Stress-2 \\
\hline Strain & 29 & 3751.12 & 43 & 3811.26 & 18 & 3750.99 & 28 & 3809.96 & 39 & 3751.62 \\
\hline Cheap & 117 & 3751.42 & 39 & 3908.92 & 59 & 3751.26 & 32 & 3901.68 & 48 & 3751.51 \\
\hline Random & 209 & 3751.12 & 61 & 3841.47 & 249 & 3756.15 & 38 & 3841.14 & 250 & - \\
\hline Random & 250 & - & 96 & 3892.18 & 191 & 3755.50 & 72 & 3891.78 & 239 & 3751.52 \\
\hline Random & 250 & - & 29 & 3852.61 & 218 & 3754.71 & 25 & 3861.31 & 225 & 3751.85 \\
\hline Random & 250 & - & 54 & 3959.23 & 250 & - & 49 & 3911.53 & 241 & 3751.58 \\
\hline Random & 214 & 3752.07 & 39 & 3898.98 & 192 & 3758.60 & 31 & 3881.71 & 212 & 3751.95 \\
\hline
\end{tabular}

Deoxyribonucleic acid, $\mathrm{g}=2$

\begin{tabular}{|l|rr|rrrr|rrrr|}
\hline & \multicolumn{4}{|c|}{ Newton } & \multicolumn{4}{|c|}{ SMACOF-I, Guttman transforms } & \multicolumn{4}{c|}{ SMACOF-I, Relaxed updates } \\
& Iter & Stress & It-1 & Stress-1 & It-2 & Stress-2 & It-1 & Stress-1 & It-2 & Stress-2 \\
\hline Strain & 26 & 3799.74 & 27 & 3800.10 & 18 & 3793.96 & 26 & 3804.24 & 16 & 3799.77 \\
\hline Cheap & 63 & 3799.21 & 13 & 3853.66 & 59 & 3798.38 & 13 & 3810.19 & 63 & 3798.28 \\
\hline Random & 250 & - & 31 & 3916.85 & 250 & - & 27 & 4011.68 & 227 & 3795.86 \\
\hline Random & 213 & 3798.99 & 28 & 3859.18 & 234 & 3793.63 & 27 & 3833.83 & 223 & 3795.03 \\
\hline Random & 250 & - & 36 & 3912.82 & 195 & 3797.02 & 30 & 3811.45 & 250 & - \\
\hline Random & 163 & 3799.14 & 39 & 3812.82 & 178 & 3792.19 & 36 & 3903.63 & 246 & 3794.56 \\
\hline Random & 250 & - & 39 & 3880.97 & 242 & 3796.10 & 33 & 3880.97 & 241 & 3795.47 \\
\hline
\end{tabular}

Deoxyribonucleic acid, $\mathrm{g}=3$

\begin{tabular}{|l|rr|rrrr|rrrr|}
\hline & \multicolumn{2}{|c|}{ Newton } & \multicolumn{9}{|c|}{ SMACOF-I, Guttman transforms } & \multicolumn{5}{|c|}{ SMACOF-I, Relaxed updates } \\
& Iter & Stress & It-1 & Stress-1 & It-2 & Stress-2 & It-1 & Stress-1 & It-2 & Stress-2 \\
\hline Strain & 20 & 5096.57 & 34 & 5100.42 & 19 & 5096.36 & 25 & 5174.04 & 18 & 5096.21 \\
\hline Cheap & 51 & 5098.66 & 17 & 5118.60 & 50 & 5098.55 & 17 & 5132.66 & 51 & 5095.01 \\
\hline Random & 233 & 5098.38 & 25 & 5138.20 & 201 & 5096.41 & 24 & 5138.20 & 215 & 5095.77 \\
\hline Random & 221 & 5097.23 & 36 & 5104.62 & 219 & 5097.86 & 33 & 5104.83 & 189 & 5097.23 \\
\hline Random & 236 & 5096.20 & 38 & 5182.18 & 192 & 5099.16 & 36 & 5182.42 & 221 & 5095.36 \\
\hline Random & 250 & - & 89 & 5144.55 & 185 & 5098.05 & 70 & 5144.55 & 250 & - \\
\hline Random & 229 & 5095.45 & 42 & 5317.46 & 250 & - & 40 & 5133.07 & 239 & 5095.37 \\
\hline
\end{tabular}


Glycopeptide antibiotic, $\mathrm{g}=1$

\begin{tabular}{|l|rc|rrrr|rrrr|}
\hline & \multicolumn{2}{|c|}{ Newton } & \multicolumn{4}{|c|}{ SMACOF-I, Guttman transforms } & \multicolumn{4}{c|}{ SMACOF-I, Relaxed updates } \\
& Iter & Stress & It-1 & Stress-1 & It-2 & Stress-2 & It-1 & Stress-1 & It-2 & Stress-2 \\
\hline Strain & 23 & 3002.20 & 31 & 3171.19 & 41 & 3002.21 & 20 & 3009.68 & 6 & 3002.84 \\
\hline Cheap & 51 & 3000.59 & 54 & 3117.53 & 112 & 3005.87 & 23 & 3136.31 & 208 & 3005.78 \\
\hline Random & 240 & 3004.27 & 27 & 3194.01 & 241 & 3005.68 & 24 & 3124.39 & 234 & 3005.11 \\
\hline Random & 216 & 3001.03 & 51 & 3183.93 & 140 & 3008.95 & 22 & 3125.97 & 144 & 3005.52 \\
\hline Random & 173 & 2999.92 & 50 & 3187.22 & 116 & 3005.34 & 22 & 3167.22 & 121 & 3008.75 \\
\hline Random & 181 & 3005.81 & 22 & 3170.75 & 157 & 3005.12 & 21 & 3113.93 & 135 & 3007.75 \\
\hline Random & 158 & 3005.60 & 29 & 3154.47 & 169 & 3006.08 & 23 & 3114.01 & 79 & 3007.74 \\
\hline
\end{tabular}

Glycopeptide antibiotic, $\mathrm{g}=2$

\begin{tabular}{|l|rr|rrrr|rrrr|}
\hline & \multicolumn{2}{|c|}{ Newton } & \multicolumn{3}{|c|}{ SMACOF-I, Guttman transforms } & \multicolumn{4}{c|}{ SMACOF-I, Relaxed updates } \\
& Iter & Stress & It-1 & Stress-1 & It-2 & Stress-2 & It-1 & Stress-1 & It-2 & Stress-2 \\
\hline Strain & 24 & 3126.98 & 42 & 3128.90 & 8 & 3126.08 & 35 & 3129.38 & 12 & 3126.71 \\
\hline Cheap & 48 & 3125.93 & 22 & 3436.67 & 11 & 3128.29 & 21 & 3436.98 & 22 & 3127.02 \\
\hline Random & 217 & 3126.75 & 23 & 3424.70 & 156 & 3127.94 & 21 & 3328.31 & 17 & 3126.40 \\
\hline Random & 167 & 3126.99 & 89 & 3329.54 & 129 & 3128.37 & 56 & 3229.72 & 18 & 3126.57 \\
\hline Random & 212 & 3126.08 & 27 & 3427.31 & 134 & 3126.94 & 22 & 3332.69 & 38 & 3126.72 \\
\hline Random & 183 & 3127.00 & 29 & 3229.37 & 181 & 3126.03 & 23 & 3231.74 & 42 & 3126.61 \\
\hline Random & 188 & 3126.18 & 21 & 3439.29 & 189 & 3126.19 & 21 & 3416.71 & 112 & 3127.10 \\
\hline
\end{tabular}

Glycopeptide antibiotic, $\mathrm{g}=3$

\begin{tabular}{|l|rc|rrrr|rrrr|}
\hline & \multicolumn{9}{|c|}{ Newton } & \multicolumn{2}{|c|}{ SMACOF-I, Guttman transforms } & \multicolumn{5}{c|}{ SMACOF-I, Relaxed updates } \\
& Iter & Stress & It-1 & Stress-1 & It-2 & Stress-2 & It-1 & Stress-1 & It-2 & Stress-2 \\
\hline Strain & 23 & 3816.61 & 37 & 3845.37 & 34 & 3815.69 & 76 & 3824.59 & 23 & 3816.15 \\
\hline Cheap & 63 & 3815.45 & 89 & 3921.57 & 89 & 3815.58 & 31 & 3921.75 & 61 & 3815.65 \\
\hline Random & 187 & 3816.91 & 29 & 3921.99 & 111 & 3815.86 & 28 & 3917.11 & 115 & 3815.57 \\
\hline Random & 191 & 3816.12 & 27 & 3923.58 & 250 & - & 18 & 3919.83 & 132 & 3815.64 \\
\hline Random & 177 & 3815.96 & 38 & 3987.99 & 153 & 3815.25 & 21 & 3896.54 & 121 & 3815.42 \\
\hline Random & 198 & $\mathbf{3 8 1 5 . 9 9}$ & 39 & 3916.14 & 162 & 3816.32 & 31 & 3917.57 & 148 & 3815.59 \\
\hline Random & 199 & 3815.63 & 37 & 3918.25 & 241 & 3815.26 & 32 & 3900.48 & 250 & \\
\hline
\end{tabular}




\section{References}

[1] J. Barzilai and J. Borwein. Two-point step size gradient methods. IMA Journal of Numerical Analysis, $8: 141-148,1988$.

[2] P. T. Boggs, R. H. Byrd, J. R. Donaldson, and R. B. Schnabel. ODRPACK: software for weighted orthogonal distance regression. ACM Transactions on Mathematical Software, 15:100-200, 1991.

[3] P. T. Boggs and J. E. Dennis. A stability analysis for perturbed nonlinear iterative methods. Mathematics of Computation, 30:1-17, 1976.

[4] P. T. Boggs, J. W. Tolle, and A. J. Kearsley. A Practical Algorithm for General Large Scale Nonlinear Optimization Problems. Technical Report NISTIR 5407, Applied and Computational Mathematics Division, National Institute of Standards and Technology, Gaithersburg, MD, 1994. To appear in SIAM Journal on Optimization.

[5] M. Browne. The Young-Householder algorithm and the least square multidimensional scaling of squared distance. Journal of Classification, 4:175-190, 1987.

[6] G. M. Crippen and T. F. Havel. Distance Geometry and Molecular Conformation. John Wiley \& Sons, New York, 1988.

[7] J. de Leeuw. Applications of convex analysis to multidimensional scaling. In J. R. Barra, F. Brodeau, G. Romier, and B. van Cutsem, editors, Recent Developments in Statistics, pages 133-145, North-Holland Publishing Company, Amsterdam, 1977.

[8] J. de Leeuw. Convergence of the majorization method for multidimensional scaling. Journal of Classification, 5:163-180, 1988.

[9] J. de Leeuw. Differentiability of Kruskal's Stress at a local minimum. Psychometrika, 49:111-113, 1984.

[10] J. de Leeuw and W. Heiser. Multidimensional scaling with restrictions on the configuration. In P. R. Krishnaiah, editor, Multivariate Analysis, North-Holland Publishing Company, Amsterdam, 1980.

[11] J. de Leeuw and W. Heiser. Theory of multidimensional scaling. In P. R. Krishnaiah and I. N. Kanal, editors, Handbook of Statistics, chapter 13, pages 285-316, North-Holland Publishing Company, Amsterdam, 1982.

[12] J. E. Dennis, D. M. Gay, and R. E. Welsch. An adaptive nonlinear least-squares algorithm. $A C M$ Transactions on Mathematical Soflware, 7:348-368, 1981.

[13] J. E. Dennis and R. B. Schnabel. Numerical Methods for Unconstrained Optimization and Nonlinear Equations. Prentice-Hall, Englewood Cliffs, New Jersey, 1983.

[14] J. C. Gilbert and C. LeMaréchal. Some numerical experiments with variable-storage quasi-Newton algorithms. Mathematical Programming, 45:407-435, 1989.

[15] W. Glunt, T-L. Hayden, S. Hong, and J. Wells. An alternating projection algorithm for computing the nearest Euclidean distance matrix. SIAM Journal on Matrix Analysis and Applications, 11:589-600, 1990.

[16] W. Glunt, T. L. Hayden, and W.-M. Liu. The embedding problem for predistance matrices. Bulletin of Mathematical Biology, 53:769-796, 1991.

[17] W. Glunt, T. L. Hayden, and M. Raydan. Molecular conformations from distance matrices. Journal of Computational Chemistry, 14:114-120, 1993.

[18] W. Glunt, T. L. Hayden, and M. Raydan. Preconditioners for distance matrix algorithms. Journal of Computational Chemistry, 15:227-232, 1994. 
[19] J. C. Gower. Some distance properties of latent root and vector methods in multivariate analysis. Biometrika, 53:315-328, 1966.

[20] P. J. F. Groenen. The Majorization Approach to Multidimensional Scaling. DSWO Press, Leiden, The Netherlands, 1993.

[21] L. Guttman. A general nonmetric technique for finding the smallest coordinate space for a configuration of points. Psychometrika, 33:469-506, 1968.

[22] T. F. Havel. An evaluation of computational strategies for use in the determination of protein structure from distance constraints obtained by nuclear magnetic resonance. Progress in Biophysics and Molecular Biology, 56:43-78, 1991.

[23] M. D. Hebden. An Algorithm for Minimization Using Exact Second Derivatives. Report TP515, Atomic Energy Research Establishment, Harwell, England, 1973.

[24] W. J. Heiser and J. de Leeuw. SMACOF-I. Technical Report UG-86-02, Department of Data Theory, University of Leiden, Leiden, The Netherlands, 1986.

[25] M. R. Hoare and P. Pal. Physical cluster mechanics: statics and energy surfaces for monatomic systems. Advances in Physics, 20:161-196, 1971.

[26] J. B. Kruskal. Multidimensional scaling and other methods for discovering structure. In K. Enslein, A. Ralston, and H. S. Wilf, editors, Statistical Methods for Digital Computers, chapter 12, pages 296-339, John Wiley \& Sons, New York, 1977. Volume III of Mathematical Methods for Digital Computers.

[27] J. B. Kruskal. Multidimensional scaling by optimizing goodness of fit to a nonmetric hypothesis. Psychometrika, 29:1-27, 1964.

[28] J. B. Kruskal. Nonmetric multidimensional scaling: a numerical method. Psychometrika, 29:28-42, 1964.

[29] J. B. Kruskal and M. Wish. Multidimensional Scaling. Sage Publications, Beverly Hills, 1978. Sage University Paper series on Quantitative Applications in the Social Sciences, 07-001.

[30] D. C. Liu and J. Nocedal. On the limited memory BFGS method for large scale optimization. Mathematical Programming, 45:503-528, 1989.

[31] M. McFarlane and F. W. Young. Graphical sensitivity analysis for multidimensional scaling. Journal of Computational and Graphical Statistics, 3:23-33, 1994.

[32] J. J. Moré. The Levenberg-Marquardt algorithm: implementation and theory. In G. A. Watson, editor, Numerical Analysis, pages 105-116, Springer Verlag, Berlin, 1977. Lecture Notes in Mathematics, 630.

[33] J. J. Moré, B. S. Garbow, and K. E. Hillstrom. User's Guide for MINPACK-1. Technical Report ANL 80-74, Argonne National Laboratories, 1980.

[34] J. A. Northby. Structure and binding of Lennard-Jones clusters: $13 \leq n \leq 147$. Journal of Chemical Physics, 87:6166-6177, 1987.

[35] M. J. D. Powell. A hybrid method for nonlinear equations. In P. Rabinowitz, editor, Numerical Methods for Nonlinear Algebraic Equations, pages 87-114, Gordon and Breach, London, 1970.

[36] M. Raydan. On the Barzilai and Borwein choice of a steplength for the gradient method. IMA Journal of Numerical Analysis, 13:321-326, 1993.

[37] R. N. Shepard. The analysis of proximities: multidimensional scaling with an unknown distance function. II. Psychometrika, 27:219-246, 1962.

[38] R. N. Shepard. The analysis of proximities: multidimensional scaling with an unknown distance function. I. Psychometrika, 27:125-140, 1962. 
[39] D. C. Sorensen. Implicit application of polynomial filters in a k-step Arnoldi method. SIAM Journal on Matrix Analysis, 13:357-385, 1991.

[40] Y. Takane, F. W. Young, and J. de Leeuw. Nonmetric individual differences multidimensional scaling: an alternating least squares method with optimal scaling features. Psychometrika, 42:7-67, 1977.

[41] P. Tarazaga and M. W. Trosset. An optimization problem on subsets of the symmetric postive semidefinite matrices. Journal of Optimization Theory and Applications, 79:513-524, 1993.

[42] W. S. Torgerson. Multidimensional scaling: I. Theory and method. Psychometrika, 17:401-419, 1952.

[43] W. S. Torgerson. Theory and Methods of Scaling. John Wiley \& Sons, New York, 1958.

[44] M. W. Trosset. The Formulation and Solution of Multidimensional Scaling Problems. Technical Report TR93-55, Department of Computational and Applied Mathematics, Rice University, Houston, TX 77251-1892, 1993. Submitted for publication.

[45] W. A. Wagenaar and P. Padmos. Quantitative interpretation of Stress in Kruskal's method multidimensional scaling technique. British Journal of Mathematical and Statistical Psychology, 24:101-110, 1971 .

[46] G. Young and A. S. Householder. Discussion of a set of points in terms of their mutual distances. Psychometrika, 3:19-22, 1938. 